\title{
Characterizing JONSWAP Rogue Waves and their Statistics via Inverse Spectral Data
}

\author{
Annalisa Calini ${ }^{\mathrm{a}}$, Constance M. Schober ${ }^{\mathrm{b}}$ \\ ${ }^{a}$ Department of Mathematics, College of Charleston, Charleston, SC 29424 USA \\ ${ }^{b}$ Department of Mathematics, University of Central Florida, Orlando, FL 32816 USA
}

\begin{abstract}
Rogue waves in random sea states modeled by the JONSWAP power spectrum are high amplitude waves arising over non-uniform backgrounds that cannot be viewed as small amplitude modulations of Stokes waves. In the context of Nonlinear Schrödinger (NLS) models for waves in deep water, this poses the challenge of identifying appropriate analytical solutions for JONSWAP rogue waves, investigating possible mechanisms for their formation, and examining the validity of the NLS models in these more realistic settings. In this work we investigate JONSWAP rogue waves using the inverse spectral theory of the periodic NLS equation for moderate values of the period. For typical JONSWAP initial data, numerical experiments show that the developing sea state is well approximated by the first few dominant modes of the nonlinear spectrum and can be described in terms of a 2- or 3-phase periodic NLS solution. As for the case of uniform backgrounds, proximity to instabilities of the underlying 2-phase solution appears to be the main predictor of rogue wave occurrence, suggesting that the modulational instability of 2-phase solutions of the NLS is a main mechanism for rogue wave formation and that homoclinic orbits of unstable 2-phase solutions are plausible models of JONSWAP rogue waves. To support this claim, we correlate the maximum wave strength as well as the higher statistical moments with elements of the nonlinear spectrum. The result is a diagnostic tool widely applicable to both model or field data for predicting the likelihood of rogue waves. Finally, we examine the validity of NLS models for JONSWAP data, and show that NLS solutions with JONSWAP initial data are described by non-Gaussian statistics, in agreement with the TOPEX field studies of sea surface height variability.
\end{abstract}

Keywords: rogue waves, JONSWAP, Nonlinear Schrödinger equation, $N$-phase solution, rogue wave diagnostics

\section{Introduction}

The Nonlinear Schrödinger (NLS) equation is one of the simplest models capturing the modulational instability and nonlinear focusing of gravity waves in deep water. Amongst its simplest solutions are periodic breathers and rational solutions over a uniform background, constructed as heteroclinic orbits (and their singular limits) of modulationally unstable Stokes waves $[1,2,6,19]$. These solutions are regarded as prototypes of for rogue waves, in that they exhibit their distinguishing features: transience, localization and steepness.

In this paper, we focus on the distinctive features of rogue waves that arise from numerical simulations of the NLS equation over a periodic spatial domain using JONSWAP initial data. Developing ocean states are described by the Joint North Sea Wave Project (JONSWAP) power spectrum (see, e.g., [18, 21]), modeling the energy distribution of waves as a function of frequency. As noted in Onorato et al. [16], realistic ocean condition are characterized by a complex spectrum whose perturbation of the carrier wave cannot be viewed as small.

Email addresses: calinia@cofc.edu (Annalisa Calini), Constance.Schober@ucf.edu (Constance M. Schober)

Preprint submitted to Elsevier

June 10, 2016

(C) 2016. This manuscript version is made available under the Elsevier user license

http://www.elsevier.com/open-access/userlicense/1.0/ 
For any given time, such realistic sea states can be converted into initial conditions for the non-dimensional NLS equation and classified in terms of their nonlinear spectrum. (For periodic boundary conditions, this is the Floquet spectrum of the spatial part of the associated Lax pair, as described in Section 2.) We confirm that JONSWAP initial data are in general far from uniform background states in that their wave forms possess nontrivial spatial structure $[13,16,19]$. Nevertheless, for spatial periods that are not too large, their nonlinear spectra are typically close to those of a certain class of 3-phase solutions of the NLS equation (see Section 2.2), and two main (easily detectable) scenarios arise: in the first, the solution is a genuine 3-phase solution that will not evolve into a rogue wave; in the second scenario, the solution is a marginally stable 3 -phase solution that is close to a heteroclinic orbit of a 2-phase solution. In all these cases, the background state cannot be regarded as either a small perturbation of the Stokes wave or a random irregular state; thus the underlying mechanism of rogue wave formation appears to be inherent to the instability of 2-phase solutions rather than the instability of a nearby uniform background.

The work presented here straddles several current directions of investigation of rogue waves in NLS models. One is the modeling of large waves over more realistic backgrounds, where we mention Osborne's analytical construction of large rogue waves from mode-coalescence of $N$-phase solutions resulting in high peaks (non-homoclinic solutions) over a non-uniform background [20]. A second is the validity of NLS solutions in realistic sea states: while our results suggest that for moderate spatial periods the more realistic JONSWAP rogue waves are close to heteroclinic orbits of 2-phase solutions of the NLS equation, an interesting question is what happens to the spatial structure of the background sea state and the mechanism and statistics of rogue wave generation for larger periods. In this context we note recent experimental and numerical work by Chabchoub et. al $[8,9]$ on the Peregrine breather (large period limit of the kind of periodic homoclinic solutions considered in this work) over an irregular domain. A third is deeper understanding of the analytical mechanism of modulational instability. While on periodic domains modulational instability can be described in terms of heteroclinic orbits of quasi-periodic states [11] (the approach taken in this work), on infinite domains this is far less well-understood. See work by Grimshaw and Tobvis [12] on the asymptotic development of breather solutions from slowly modulated Stokes waves, and more recent work by Biondini and Mantzavinos [5].

The rest of the paper is organized as follows: In Section 2, we consider a family of 3-phase solutions of the NLS equation that can be taken as a simple model of a modulationally unstable non-uniform background of the kind observed in JONSWAP initial data. We use a Bäcklund transformation to construct its heteroclinic orbits, compare their dynamics with that of JONSWAP data near rogue-wave status, and propose them as candidates for JONSWAP rogue waves. In Section 3 we present the numerical results showing evidence that JONSWAP rogue waves are close to heteroclinic orbits of 2-phase solutions of the NLS equation, thus extending the use of inverse spectral theory to a wider range of models of rogue waves. As for the uniform background cases, we show that proximity to heteroclinic structures of the more complex backgrounds correlates with increased probability of rogue wave generation. We also discuss how the NLS spectral diagnostic tool performs well in predicting the likelihood of rogue waves also for JONSWAP initial data that evolve by a higher order NLS (HONLS) equation, providing a more accurate description of wave motion. Finally, in subsection 3.3 we compare JONSWAP initial data evolving by both the NLS and HONLS equations

to field data, showing a reasonable qualitative match in their non-Gaussian statistics, but over-prediction of rogue wave events by the NLS and HONLS models.

\section{Characterization of rogue waves for JONSWAP initial data}

\subsection{The Focusing Nonlinear Schrödinger Equation framework}

The governing equation is given in dimensionless form by the focusing cubic Nonlinear Schrödinger (NLS) equation

$$
i u_{t}+u_{x x}+2|u|^{2} u=0
$$

over a spatially periodic domain. In order to model rogue waves over non-uniform backgrounds, we shall represent the background sea state by a modulationally unstable $N$-phase periodic potential $u(x+L, t)=$ 
$u(x, t)$ over an extended domain $x \in\left[-\frac{n L}{2}, \frac{n L}{2}\right]$, where $n \in \mathbb{N}$ is chosen so that $u$ has one or more linear unstable modes, as described below in detail.

Recall that the NLS equation (1) is the compatibility condition of the AKNS system, after the authors of $[3]$ :

$$
\begin{aligned}
\mathcal{L}(u) \vec{\psi} & =\lambda \vec{\psi} \\
\vec{\psi}_{t} & =\left(\begin{array}{cc}
-2 \mathrm{i} \lambda^{2}+\mathrm{i}|u|^{2} & 2 \mathrm{i} \lambda u-u_{x} \\
2 \mathrm{i} \lambda \bar{u}+\bar{u}_{x} & 2 \mathrm{i} \lambda^{2}-\mathrm{i}|u|^{2}
\end{array}\right) \vec{\psi},
\end{aligned}
$$

comprising the matrix eigenvalue problem for the linear operator

$$
\mathcal{L}(u)=\mathrm{i} \sigma_{3} \frac{\partial}{\partial x}+\left(\begin{array}{cc}
0 & u \\
-\bar{u} & 0
\end{array}\right)
$$

and a linear equation for the time evolution of the eigenfunction $\vec{\psi} \in \mathbb{C}$.

Given a periodic potential $u$, the Floquet spectrum $\sigma(u)$ of $\mathcal{L}(u)$ characterizes the level set of $u$ and of its stable and unstable manifolds. In particular, linear instabilities are usually associated with complex points of multiplicity two (complex double points) in the discrete component of $\sigma(u)$.

The Floquet spectrum of $u$ can be computed explicitly by solving the eigenvalue problem $(2)$ at $u(x, 0)$, given the well-known fact that the spectrum is invariant under the NLS evolution [15]. In terms of the Floquet discriminant

$$
\Delta(u ; \lambda)=\operatorname{Trace}\left(\Psi(x+L, t ; \lambda) \Psi(x, t ; \lambda)^{-1}\right),
$$

where $\Psi(x, 0 ; \lambda)$ is a matrix whose columns are linearly independent eigenfunctions, the Floquet spectrum is defined as the set of complex $\lambda$ 's for which the eigenfunctions of $\mathcal{L}(u)$ are bounded in $x$, i.e.

$$
\sigma(u)=\{\lambda \in \mathbb{C} \mid \Delta(u ; \lambda) \in \mathbb{R},-2 \leq \Delta \leq 2\} .
$$

Complex double points are zeros of $\Delta^{2}(\lambda)-4$ of multiplicity two, i.e. satisfying $\Delta= \pm 2, \mathrm{~d} \Delta / \mathrm{d} \lambda=0$, $\mathrm{d}^{2} \Delta / \mathrm{d} \lambda^{2} \neq 0$, and are generically located within (complex) bands of continuous spectrum.

$N$-phase (or finite-gap) potentials are solutions of the form $u\left(\theta_{1}, \ldots, \theta_{N}\right), \theta_{i}=k_{i} x+\omega_{i} t$, that are periodic in each phase, and as such periodic in $x$ for appropriately selected wave numbers, and quasi-periodic in time. The Floquet spectrum of an $L$-periodic $N$-phase potential is characterized by a finite number of bands of in the complex plane (each associated with a particular phase) and, if $u$ is regarded as a solution of the initialboundary value problem (1) over a domain of length $n L, n \in \mathbb{N}$, then the associated spectrum will contain complex double points (the more the larger $n$ is), reflecting the modulational instability (i.e. instability with respect to long-wavelength perturbations) of such potentials.

Important Observation: Since the Floquet spectrum of a modulationally unstable $N$-phase potential $u$ defines the level set of $u$ in phase space, it also coincides with the Floquet spectrum of its homoclinic or heteroclinic orbits (namely its stable and unstable manifolds). Such heteroclinic orbits can be used to model rogue waves over the background $u$ in much the same way as periodic breathers of modulationally unstable Stokes waves have been widely used to model rogue waves over uniform backgrounds. In fact we will argue that JONSWAP rogue waves can be well-approximated by heteroclinic orbits of unstable $N$-phase potentials and that proximity to such structures correlates with likelihood of rogue wave formation.

For the present work, we shall take the non-uniform background $u$ to be the following finite-gap solution first derived in [4]:

$$
u(x, t)=\frac{\chi}{2} \frac{A(x) \operatorname{cn}(t, \chi)+\mathrm{i} \sqrt{1+\chi} \operatorname{sn}(t, \chi)}{\sqrt{1+\chi}-A(x) \operatorname{dn}(t, \chi)} \mathrm{e}^{\mathrm{i} t}, \quad \text { with } A(x)=\frac{\operatorname{cn}\left(\sqrt{\frac{1+\chi}{2}} x, \sqrt{\frac{1+\chi}{1+\chi}}\right)}{\operatorname{dn}\left(\sqrt{\frac{1+\chi}{2}} x, \sqrt{\frac{1+\chi}{1+\chi}}\right)} .
$$


Formula (6) describes a 2-phase standing wave arising as the degeneration of a 3 -phase solution due to symmetry in its spectrum. ${ }^{1}$ This solution is periodic in space of period $L=4 \sqrt{2} \frac{K(m)}{\sqrt{1+\xi}}$, where $m=\sqrt{\frac{1+\chi}{1+\chi}}$, and also time-periodic with temporal period $T=4 \sqrt{2} K(\chi)$.
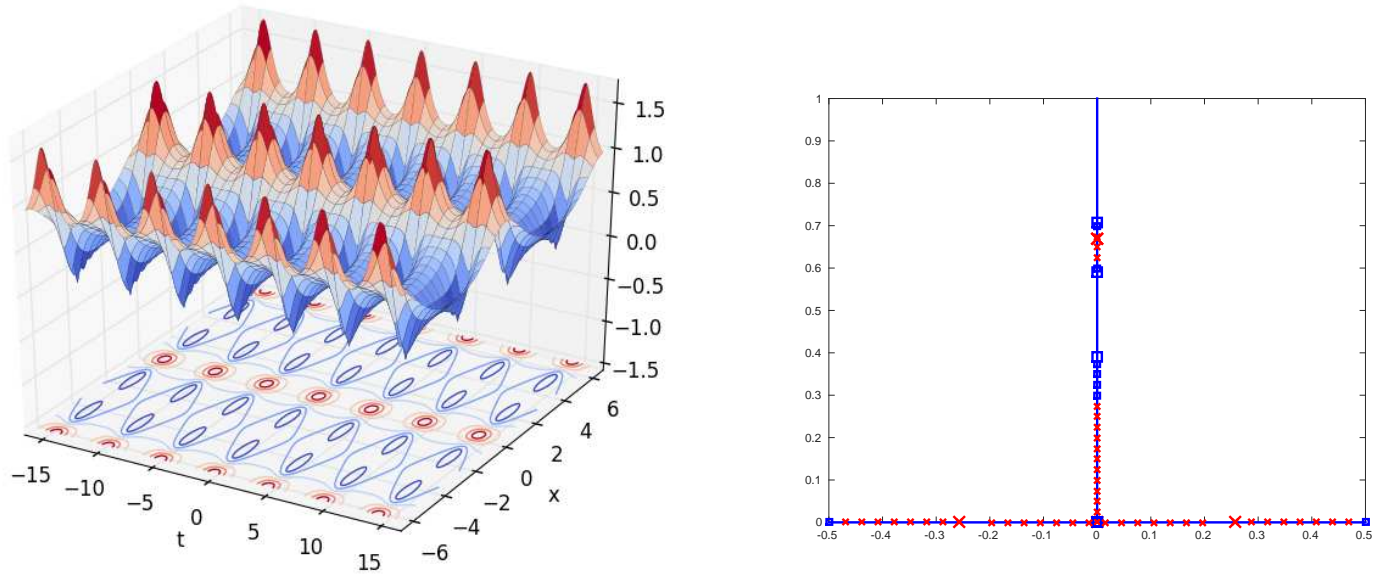

Figure 1: (left) Surface and contour plot of $|u(x, t)|$ over two periods in $x$, (right) Floquet spectrum $\sigma(u)$ with a band of imaginary spectrum containing one large gap and one pure imaginary complex double point $\nu$ (large red cross) in the upper half plane. $(\chi=0.92, L=6.4812, \nu=\mathrm{i} 0.6689)$

The amplitude and contour plots of $u(x, t)$ over a spatial interval of length $2 L$ are shown in Fig. 1(left), clearly displaying its doubly-periodic structure. As a solution of the NLS equation (1) over the extended domain, it is linearly unstable with one unstable mode, as reflected in its Floquet spectrum $\sigma(u)$.

Figure 1(right) shows the component of $\sigma(u)$ within the upper half plane. The continuous spectrum consists of the real axis together with a band of imaginary spectrum containing a single large gap. The sole imaginary double point is indicated by a large red cross in the top part of the band of imaginary spectrum. Small blue squares and small red crosses label points of continuous spectrum where the Floquet discriminant is real and positive, and real and negative (respectively). Finally, large crosses and large squares label points of the discrete (periodic) spectrum.

This particular solution was chosen because its Floquet spectrum is qualitatively comparable to the limiting configuration of the Floquet spectrum associated with typical rogue wave JONSWAP data, but with the additional symmetry $\lambda \rightarrow-\lambda$, making the construction of heteroclinic orbits and their visualization (as standing waves) simpler. The reader can compare Fig. 1(right) and Fig. 3(middle); the latter shows the Floquet spectrum of a JONSWAP rogue wave, containing a large gap in top part of the complex band of continuous spectrum, a very small gap in the bottom part, and a small band of complex spectrum off the real axis. This spectral configuration approximates the Floquet spectrum of the heteroclinic orbit of a degenerate 3-phase solution of the same type as (6), differing only in which of the two unstable modes has been excited.

Before constructing the corresponding heteroclinic orbits, we briefly review JONSWAP rogue waves.

\footnotetext{
${ }^{1}$ For the NLS equation, the number $N$ of phases of a finite-gap solution is related to the number $2 g+2$ of branch points via the formula $N=g+1$ (here $\mathrm{g}$ is the genus of the associated Riemann surface). The Floquet spectrum of (6) has 6 branch points in the complex plane, however, the extra symmetry $\lambda \rightarrow-\lambda$ results in a linear relation among the three phases, so the solution degenerates in a 2-phase solution.
} 


\subsection{JONSWAP solutions of the NLS equation}

We next present initial conditions for the NLS equation (1) for random sea states described by the Joint North Sea Wave Project (JONSWAP) spectrum in dimensionless form [14, 21]:

$$
\begin{aligned}
S(f) & =\frac{\alpha}{f^{5}} \mathrm{e}^{-\frac{5}{4} f^{-4}} \gamma^{r}, \\
r & =\mathrm{e}^{-\frac{1}{2}\left(\frac{f-1}{\sigma}\right)^{2}}, \quad \sigma= \begin{cases}0.07 & f \leq 1 \\
0.09 & f>1\end{cases}
\end{aligned}
$$

The JONSWAP spectrum models the energy distribution of waves in the ocean in terms of the frequency $f$, normalized with respect to the dominant frequency determined by wind speed (leading to enhanced nonlinear interactions). The parameter $\alpha$ is related to the amplitude and energy content of the wave field (waves continue to grow as specified by $\alpha$ ), while $\gamma$ is related to peakedness with $S(f)$ becoming narrower about the dominant peak as $\gamma$ increases.

Developing sea states are governed by $S(f)$ for a suitable range of $\alpha$ and $\gamma$ values. Typical ranges are $0.008<\alpha<0.02$ and $2<\gamma<8$ (the latter determined by the ratio of the coefficients of the nonlinear and dispersive terms in the dimensional form of the NLS equation, the so-called Ursell number).

Remark: Note that the physical space and time coordinates $\left(x_{p}, t_{p}\right)$ for the deep water NLS model in dimensional form are given by, respectively, $x_{p}=c t$ and $t_{p}=a x+b t$, where the parameters $a, b, c$ are introduced to nondimensionalize the equation. Thus, the initial condition at $t=0$ of equation (1) should be interpreted as the boundary condition at $x_{p}=0$ for a laboratory experiment.

At $t=0$ the water surface elevation can be modeled as a random wave process of the form [17]

$$
\eta(x, 0)=\sum_{n=1}^{N} C_{n} \cos \left(2 \pi k_{n} x-\phi_{n}\right), \quad x \in[-L / 2, L / 2] .
$$

Here $C_{n}=\sqrt{2 S\left(k_{n}\right) \Delta k}$ and $k_{n}=n \Delta k=n / L, n=1, \ldots, N$. The random phases $\phi_{n}$ are assumed to be uniformly distributed on $(0,2 \pi)$.

From the dimensionless surface elevation $\eta(x, 0)$, one reconstructs the NLS solution $u(x, 0)$ at $t=0$,the complex amplitude of a slowly modulated nonlinear wave train in deep water-, using the relationship $\eta(x, 0)=\epsilon / \sqrt{2} \operatorname{Re}\left\{u(x, 0) e^{2 \pi i k_{c} x}\right\}$, where $k_{c}$ is the wave number of the carrier wave [22].

Thus we can compute the Hilbert transform of $\eta(x, 0)$, and interpret it as the conjugate function of an associated analytic function, to obtain the JONSWAP initial condition for equation (1),

$$
u(x, 0)=\frac{\sqrt{2}}{\epsilon} \sum_{n=1}^{N} C_{n} e^{i\left(2 \pi\left(k_{n}-k_{c}\right) x-\phi_{n}\right)}, \quad x \in[-L / 2, L / 2] .
$$

As the resulting NLS solution $u(x, t)$ evolves in time, we monitor the strength function

$$
S(t):=\frac{\max _{x}|u(x, t)|}{H_{s}(t)}
$$

where $H_{s}(t)$ is the significant wave amplitude, defined as four times the standard deviation of the surface amplitude.

Rogue wave criterion. (Heuristic) A rogue wave occurs at $t^{*}$ if $S\left(t^{*}\right)>2.2$.

Figures 2(left) and 3(left) show the Floquet spectrum for JONSWAP data with $\gamma=4$ and $\alpha=0.012$ for two different sets of randomly generated phases $\left\{\phi_{n}\right\}_{n=1}^{N}$. In the numerical experiments, $N$ (or, equivalently, the cut-off frequency) is a large positive integer related to the number of Fourier modes used in the numerical scheme. 
The resulting Floquet spectra have two gaps near the imaginary axis (the small spines emanating from the real axis can be neglected), corresponding to 3-phase solutions that may be stable (Fig. 2(left)) or marginally stable, that is close to unstable 2-phase solutions whose spectra possess one complex double point (Fig. 3(left)). This type of spectral configuration occurs generically in the numerical experiments for any choice of $\alpha, \gamma$, and the random phases, where varying these parameters only results in changing the gap sizes.

As shown in Section 3, a Floquet spectrum with two large gaps typically corresponds to a non-rogue wave realization, one for which $S(t)<2.2, \forall t$ (Fig. 2 (middle-right)), while a Floquet spectrum with one large gap and one small gap is typically associated with a rogue wave realization for which $S\left(t^{*}\right)>2.2$ at one or more times $t^{*}$ (Fig. 3 (middle-right)).

Remark. The initial phases are as important as the energy $\alpha$ and peakedness parameter $\gamma$ in determining the strength of the wave field, i.e. whether the resulting wave form will or will not become a rogue wave. In fact, for fixed $\alpha$ and $\gamma$, different sets of randomly generated phases can result in very different behaviors. Because of this, the Floquet spectrum of JONSWAP data is a superior diagnostic tool than just using $\alpha$ and $\gamma$.

Since rogue waves occur when the second gap is small, in other words near a state where the gap has collapsed to a complex double point, we can interpret JONSWAP rogue waves as being close to heteroclinic orbits of 2-phase solutions of the NLS equation.
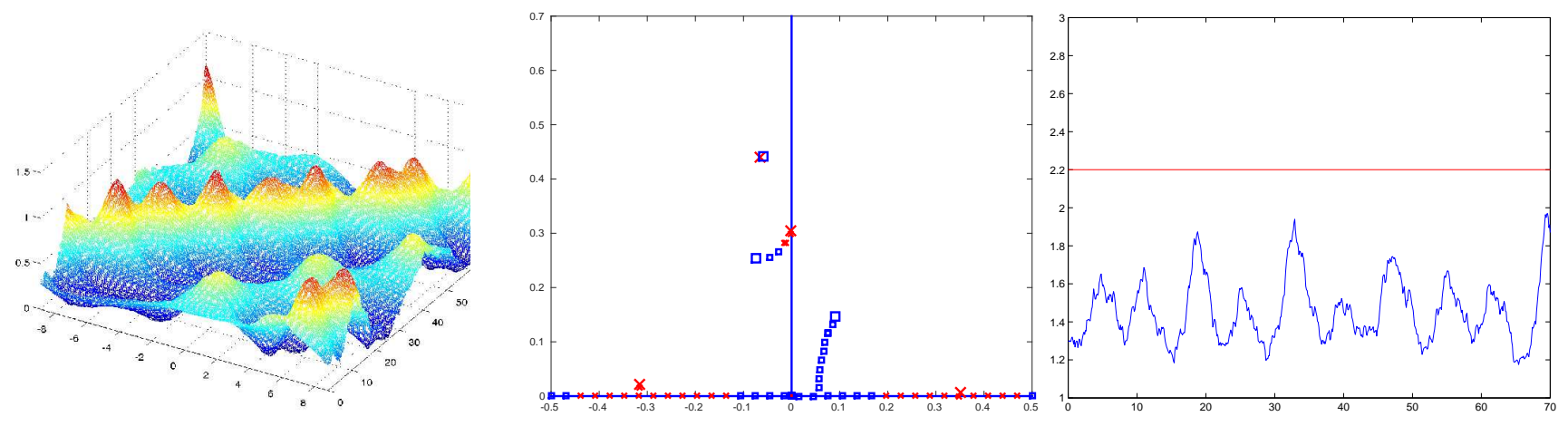

Figure 2: Non-rogue wave realization: (left) amplitude plot of $|u(x, t)|$, (middle) nonlinear spectrum far from homoclinic data, and (right) evolution of $S(t)$ for JONSWAP data $(\gamma=4, \alpha=0.012)$ for $0<t<70$.
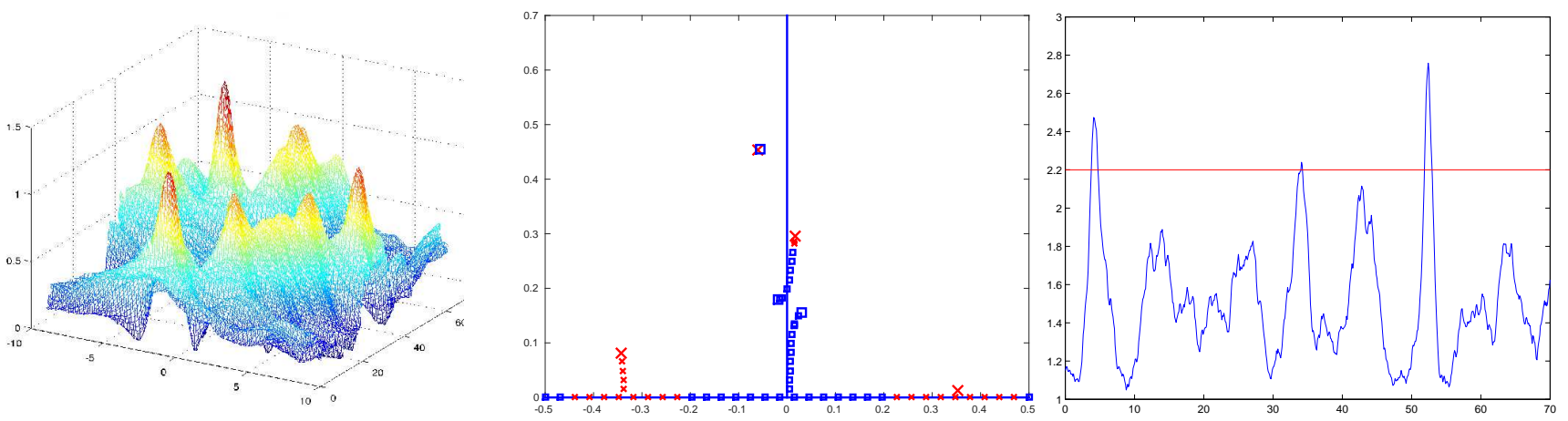

Figure 3: Rogue wave realization: (left) amplitude plot of $|u(x, t)|$, (middle) nonlinear spectrum close to homoclinic data, and (right) evolution of $S(t)$ for JONSWAP data $(\gamma=4, \alpha=0.012)$ for $0<t<70$. 


\subsection{Constructing heteroclinic orbits}

A Bäcklund transformation based at a complex double point of an unstable periodic $N$-phase potential produces a heteroclinic orbit, i.e. a solution that limits to two different $N$-phase potentials on the same level set as the seed potential as $t \rightarrow \pm \infty$.

We employ an approach developed in [23], based on first constructing a gauge transformation that gives a dressing (or Darboux) transformation of the eigenfunction associated with the seed potential, and then algebraically deriving a formula for the heteroclinic solution associated with the dressed eigenfunction.

Given an NLS solution $u$ and a fixed value $\nu$ of the spectral parameter, let $\vec{\psi}$ be a nontrivial solution of the AKNS system at $(u, \nu)$. Then

$$
u_{h}=u-2(\nu-\bar{\nu}) \frac{\psi_{1} \bar{\psi}_{2}}{\left|\psi_{1}\right|^{2}+\left|\psi_{2}\right|^{2}}
$$

is the Bäcklund transformation of $u$ based at $\nu$.

Moreover, the gauge matrix

$$
G=N\left(\begin{array}{cc}
\lambda-\nu & 0 \\
0 & \lambda-\bar{\nu}
\end{array}\right) N^{-1}, \quad \text { with } \quad N=\left(\begin{array}{cc}
\psi_{1} & -\bar{\psi}_{2} \\
\psi_{2} & \bar{\psi}_{1}
\end{array}\right)
$$

transforms a solution $\vec{\phi}(x, t)$ of the AKNS system at $(u, \lambda)$ to an eigenfunction $\vec{\phi}_{h}(x, t)=G(x, t) \vec{\phi}(x, t)$ at $\left(u_{h}, \lambda\right)$.

Note that we must have $\nu \notin \mathbb{R}$ to obtain a nontrivial transformation. Also, because $u_{h}$ is expressed in terms of quadratic products of components of $\vec{\psi}, u_{h}$ is periodic only if $\nu$ is a point of the discrete spectrum. When $u$ is an $N$-phase potential, it can be shown that if $\vec{\psi}$ is chosen to be one of the Bloch eigenfunctions or if $\nu$ is an endpoint of a band of spectrum, then $u_{h}$ is a torus translate of $u$, i.e. obtained from $u$ by constant shifts in its phases.

To obtain a heteroclinic orbit of (6), we compute two linearly independent solutions $\vec{\psi}^{ \pm}$of the AKNS system (2),(3) at $(u, \nu)$ and $x \in[-L, L]$, where $\nu$ is the imaginary double point in the upper half plane, and construct a nontrivial eigenfunction of the form

$$
\vec{\psi}(x, t ; \nu)=\vec{\psi}^{+}(x, t ; \nu)+c \vec{\psi}^{-}(x, t ; \nu), \quad c \in \mathbb{C} .
$$

The Bäcklund transformation formula (8) yields a heteroclinic orbit for any value of $c \in \mathbb{C}$. Figures $4-5$ and $6-7$ describe the resulting solutions for two different choices of the parameter $c$. Writing $c=\rho \mathrm{e}^{i \beta}$, the phase $\beta$ is seen to be related to spatial separation of the modes of the heteroclinic solution.

Case: $\rho=0, \beta=0$. This choice of $\beta$ corresponds to a solution with spatially coalesced modes (Fig. 5 (left)) that rises steeply to a maximum amplitude of about 3. Its shape is that of a classical rogue wave (single humped, localized in both space and time), with wave strength well into the rogue wave regime (Fig. 4(right)). 

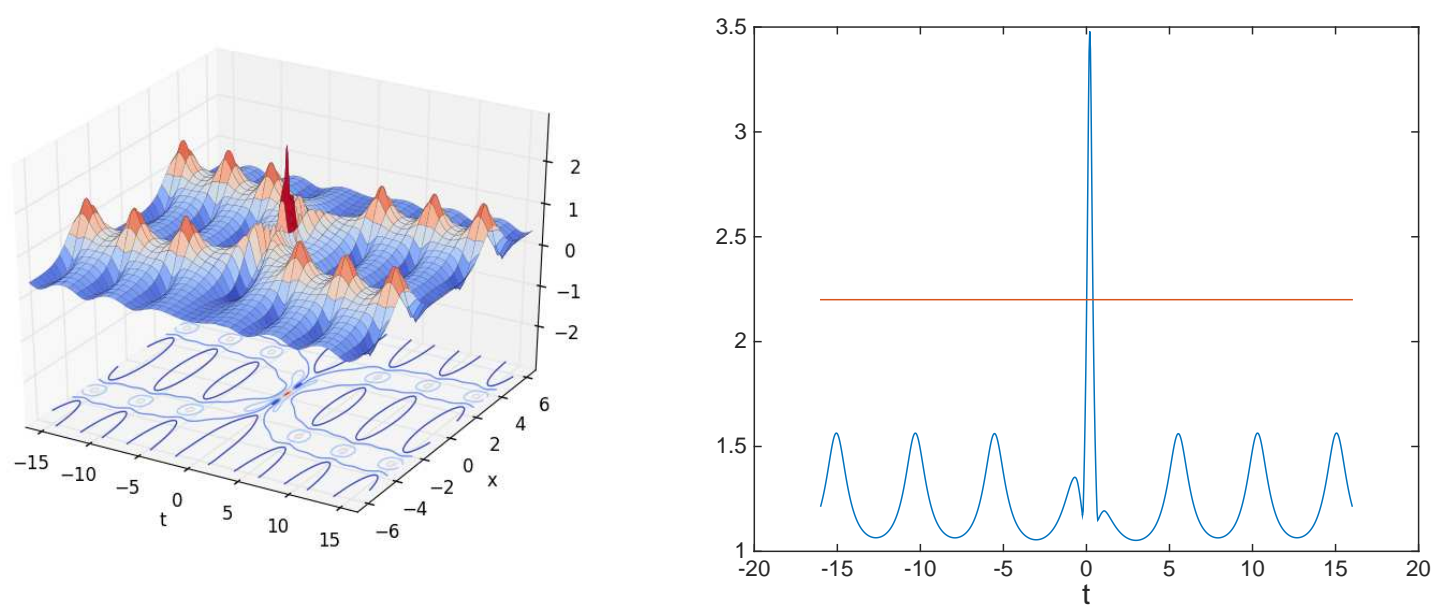

Figure 4: A (heteroclinic) rogue wave over $u$ with coalesced central peak: (left) surface and contour plot of $\left|u_{h}(x, t)\right|,($ right) evolution of $S(t) .(\rho=0, \beta=0$. $)$
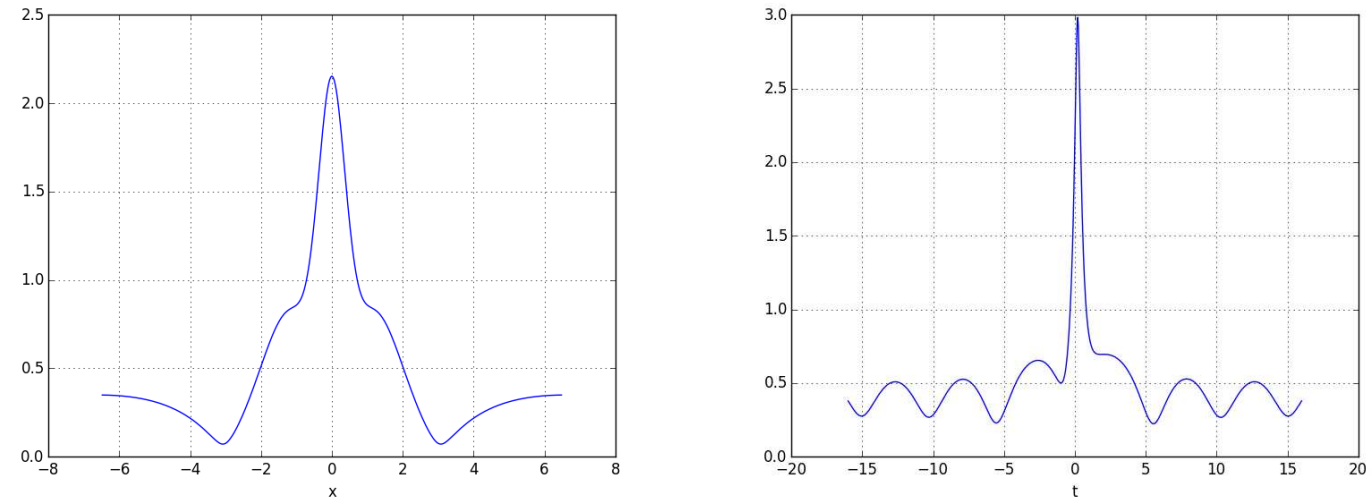

Figure 5: Coalesced case: (left) Initial profile $\left|u_{h}(x, 0)\right|$, (right) time trace of $\left|u_{h}(0, t)\right|(\rho=0, \beta=0$.)

Case: $\rho=0, \beta=\frac{\pi}{3}$. This choice of $\beta$ corresponds to a wave form that is initially in a non-fullycoalesced state (Fig. 7(left)) and rises steeply to a maximum amplitude of about 2.6 (significantly lower than in the coalesced case). The central peak has additional spatial structure as indicated by the contour plot (Fig. 6(left)), that appears to be related with to lesser steepness and height. Yet, its wave strength is also well into the rogue wave regime (Fig. 6(right)) 

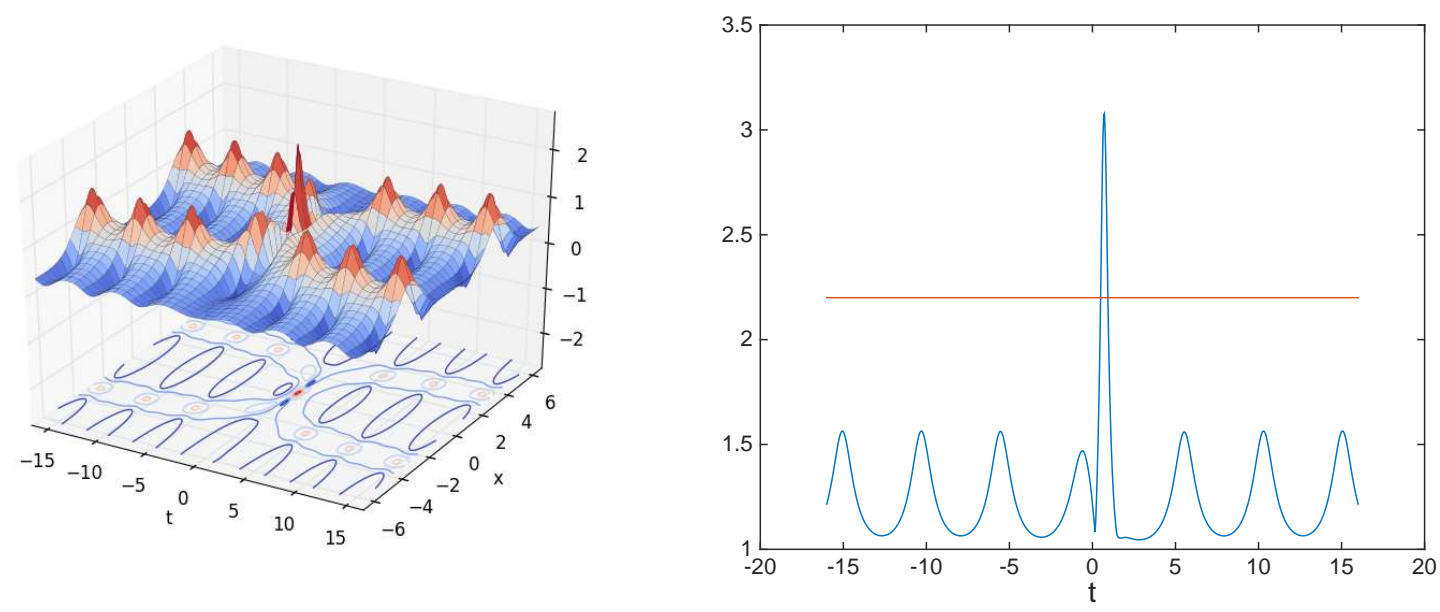

Figure 6: A (heteroclinic) rogue wave of $u$ with non-coalesced central peak: (a) Surface and contour plot of $\left|u_{h}(x, t)\right|,(\mathrm{b})$ evolution of $S(t) .\left(\rho=0, \beta=\frac{\pi}{3}.\right)$
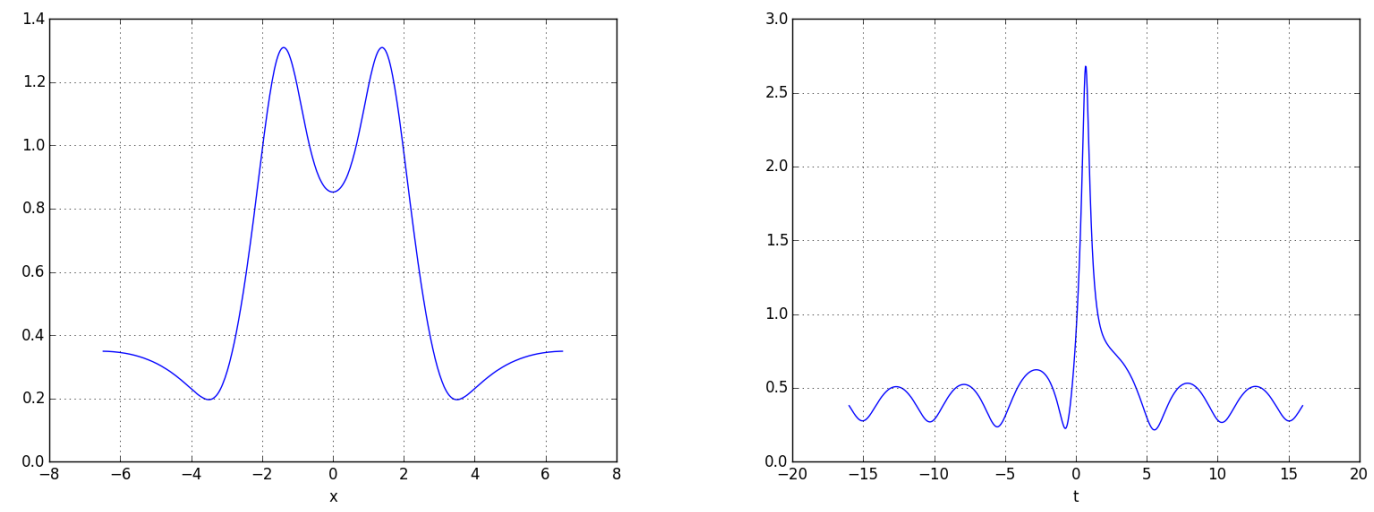

Figure 7: Non-coalesced case: (left) Initial profile $\left|u_{h}(x, 0)\right|$, (right) time trace of $\left|u_{h}(0, t)\right|\left(\rho=0, \beta=\frac{\pi}{3}\right.$.)

\section{Numerical Experiments}

In this section we investigate numerically the generation of rogue waves for sea states characterized by the JONSWAP spectrum. We use three approximations to the wave dynamics: 1) the NLS equation, 2) a more accurate model given by a higher order extension (HONLS) of the NLS equation, and 3) a linearly damped HONLS model suggested by comparison with field data (Section 3.3).

The three models are obtained from the following governing equation for the complex envelope $u(x, t)$ of the wave train

$$
\mathrm{i} u_{t}+u_{x x}+2|u|^{2} u+\mathrm{i} \Gamma u+\mathrm{i} \epsilon\left(\frac{1}{2} u_{3 x}-8|u|^{2} u_{x}-2 u i\left[\mathcal{H}|u|^{2} \mid\right]_{x}\right)=0
$$

where $0 \leq \epsilon \ll 1$ and $\Gamma \geq 0$ and $\mathcal{H}[f](x)=\frac{1}{\pi} \int_{-\infty}^{\infty} \frac{f(\xi)}{x-\xi} d \xi$ is the Hilbert transform of $f$, for the following parameter choices: 1) $\epsilon=\Gamma=0$ (NLS), 2) $\epsilon \neq 0, \Gamma=0$ (HONLS), 3) $\epsilon \neq 0, \Gamma \neq 0$ (HONLS with linear damping). Equation (9) will be considered on spatially periodic domains. 
As initial conditions for the NLS and HONLS equations we consider three large ensembles, each consisting of 640 random realizations of JONSWAP data (7) with $\gamma=2, \ldots, 8, \alpha=0.012,0.016$ and randomly generated phases. The numerical domain of the simulations is $L=4 \sqrt{2} \pi$ or approximately 60 wavelengths in space. Requiring periodic boundary conditions, equation (9) is integrated using a fourth-order exponential time differencing integrator [10] with 256 Fourier modes in space and a fourth-order Runge-Kutta discretization in time $\left(\Delta t=10^{-3}\right)$. The Floquet spectrum of initial the data is computed by the direct spectral transform, by solving system (2) constructing the discriminant $\Delta$ and computing the zeros of $\Delta \pm 2$ using a root solver based on Muller's method [11]. The spectrum is calculated with an accuracy of $\mathcal{O}\left(10^{-6}\right)$, whereas the spectral quantities we are interested in range from $\mathcal{O}\left(10^{-3}\right)$ to $\mathcal{O}\left(10^{-1}\right)$.

Under perturbations of the initial condition, a complex double point will typically split into two simple points $\lambda_{ \pm}$, thus opening a gap in the band of spectrum. The splitting distance $\delta\left(\lambda_{+}, \lambda_{-}\right)=\left|\lambda_{+}-\lambda_{-}\right|$is thus used to measure closeness in spectral space of the given initial data to homoclinic data.

The numerical experiments consistently demonstrate that: 1) the spectral measure $\delta$ is an effective diagnostic tool for determining the likelihood of rogue waves and 2) the development of rogue waves in numerical experiments for all three models is correlated with the proximity to heteroclinic orbits of 2-phase solutions of the NLS equation.

\subsection{Dependence of the maximum strength on the Floquet spectral data}

We first explore the relation between the maximum strength $S_{\max }$ of the wave field and the higher statistical moments, specifically skewness $S k e w$ and (excess) kurtosis $K$ defined below:

$$
\operatorname{Skew}(|u|):=\frac{\mu_{3}}{\sigma^{3}}, \quad K(|u|)=\frac{\mu_{4}}{\sigma^{4}}-3
$$

where

$$
\mu_{i}(|u|):=\sum_{j=1}^{N} \frac{\left(\left|u_{j}\right|-|\bar{u}|\right)^{i}}{N},
$$

$\sigma=\left(\mu_{2}\right)^{1 / 2}$ is the standard deviation, $|\bar{u}|$ is the mean amplitude of the wavefield, and $N$ is the number of data points sampled.

Skewness and kurtosis are measures of the shape of the distribution of the wave field and help characterize the variability of the data set. Skewness measures the asymmetry of the probability distribution function (PDF). Excess kurtosis measures the peakedness of the PDF relative to a Gaussian distribution. A high kurtosis indicates increased probability of rogue waves as the contribution of the outliers to the distribution becomes significant.

In Figure 8 each circle represents the maximum strength $S_{\max }=\max _{t \in[0,20]} S(t)$ achieved during one simulation of the NLS equation (1) as a function of: initial $K$ (top left), maximum kurtosis $K_{\max }$ (top right), initial Skew (bottom left), maximum skewness Skew $\max$ (bottom right). The rogue wave threshold is indicated by the line $S_{\max }=2.2$.

The plots show that initial Skew and $K$ are unsuitable for predicting rogue wave formation, since there is no clear relationship between their values and the maximum strength $S_{\max }$. On the other hand, $S_{\max }$ noticeably increases as $S k e w_{\max }$ and $K_{\max }$ increase (Fig 8(top and bottom left)). However, skewness and kurtosis both increase as the strength of the wave field increases and the maxima of $S(t), K(t)$, and $S k e w(t)$ occur at approximately the same time. By the time the maxima of the skewness and kurtosis are achieved, the rogue wave is already forming. For this reason, from a practical standpoint, $K_{\max }$ and $S k e w_{\max }$ are not useful as a predictive tool for the maximum strength. In principle, to obtain a predictive tool, one would like to be able to estimate $S_{\max }, K_{\max }$, and $S k e w_{\max }$ in terms of the initial data alone, without requiring any knowledge of the time evolution of the sea surface.

In Section 2, examples of JONSWAP waves and the associated Floquet spectra (Figures 2(left) and 3 (left)) suggest an intriguing correlation between proximity to homoclinic data and occurrence of rogue waves. Consistently, JONSWAP initial data have similar 2-gap spectral configurations, where the size of the first gap (near the top part of the complex band of continuous spectrum) remains large regardless of the 

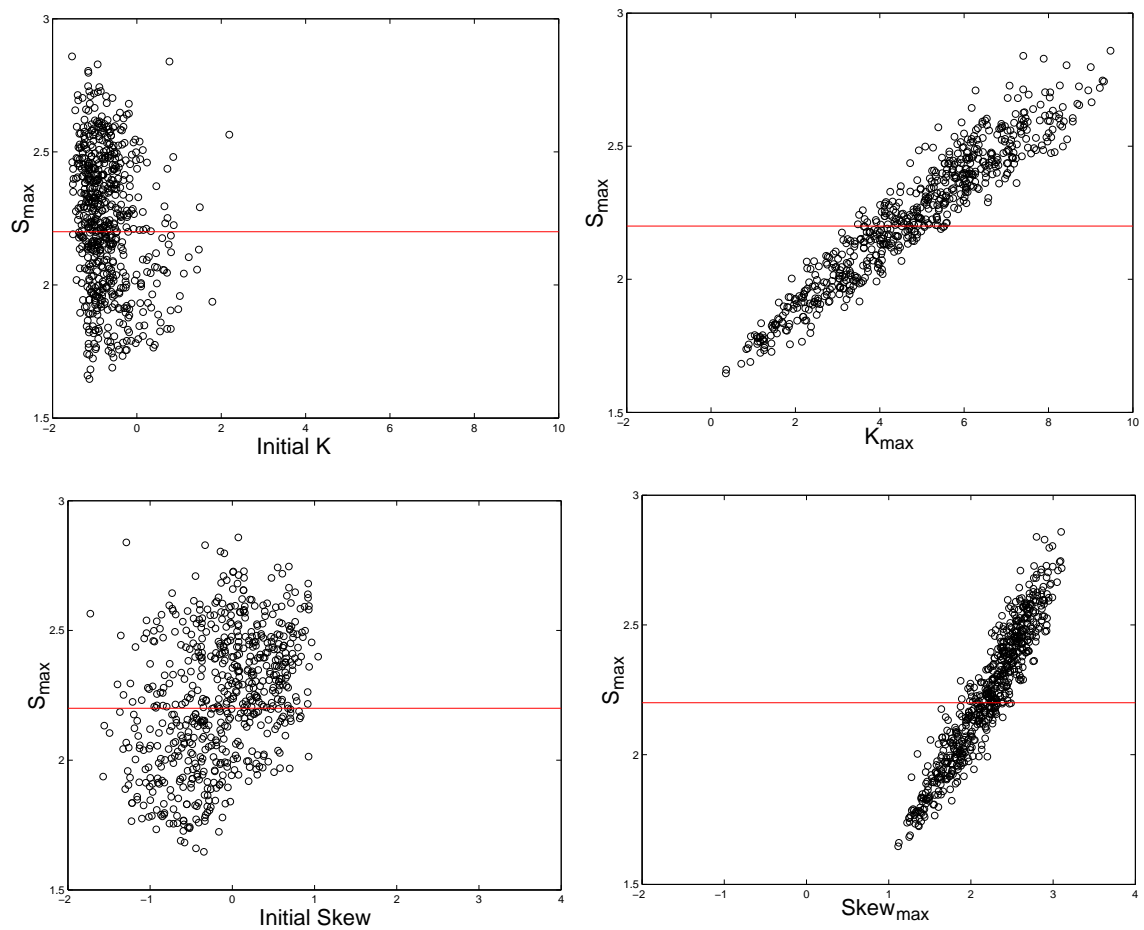

Figure 8: $S_{\max }$ as a function of: (top left) initial kurtosis, (top right) maximum kurtosis, (bottom left) initial skewness, (bottom right) maximum skewness for the NLS equation (1).

values of $(\gamma, \alpha)$ and the choice of random phases. However, the size of the second gap varies considerably for different realizations of the random phases. As a result, for JONSWAP data, the splitting distance $\delta\left(\lambda_{+}, \lambda_{-}\right)=\left|\lambda_{+}-\lambda_{-}\right|$refers to the second gap.

Figure 9(left) presents the outcomes of an ensemble of experiments the NLS equation with randomly generated JONSWAP initial data. Each circle represents the maximum strength, $S_{\max }=\max _{t \in[0,20]} S(t)$, attained during one simulation, as a function of the splitting distance $\delta$. The rogue wave threshold is again indicated by the line $S_{\max }=2.2$.

Significantly, $S_{\max }$ is a decreasing function of $\delta$, so the closer the initial condition is to instabilities (i.e. the smaller $\delta$ ), the higher the maximum strength. (Note that there are fewer data points when $\delta$ is very small or very large, so a trend in these regions cannot be clearly established.) The solid curve in Figure 9 (left) represents the bin averaged $S_{\max }$ obtained using 15 uniform bins in $\delta$. As $\delta$ increases the bin averaged strength decreases until at $\delta \approx 0.15$ the bin average $S_{\max }$ begins to fall below the rogue wave threshold. As $\alpha$ and $\gamma$ are varied the behavior is robust, with qualitatively similar plots of $S_{\max }$ vs $\delta$ regardless of the chosen $(\gamma, \alpha)$. Thus the spectral measure $\delta$ is a good predictor of $S_{\max }$ and rogue wave occurrence.

Alternate representations of the outcomes of the NLS experiments are provided in Figure 10. The histogram in Fig 10(left) bins the data according to the maximum strength and gives the total number of rogue waves from the entire ensemble with maximum strength prescribed by the bin. Overlaying this histogram is the average splitting distance as a function of $S_{\max }$, where the average is taken over all waves within the bin. In general, as the maximum strength increases the splitting distance decreases. In contrast, Figure 10(right) bins the data with respect to $\delta$ and gives the probability $\mathcal{P}\left(\delta_{b i n}\right)$ that a wave within the given bin is a rogue wave. As $\delta$ decreases $\mathcal{P}\left(\delta_{\text {bin }}\right)$ increases. From a practical standpoint, $\mathcal{P}\left(\delta_{b i n}\right)$ provides more relevant information than the histogram. For example, if $\delta<0.1, \mathcal{P}\left(\delta_{b i n}\right)>75 \%$ and a rogue wave is likely; while if $\delta>0.2$ then, there is a low probability $\left(\mathcal{P}\left(\delta_{\text {bin }}\right)<20 \%\right)$ that a rogue wave will occur. 

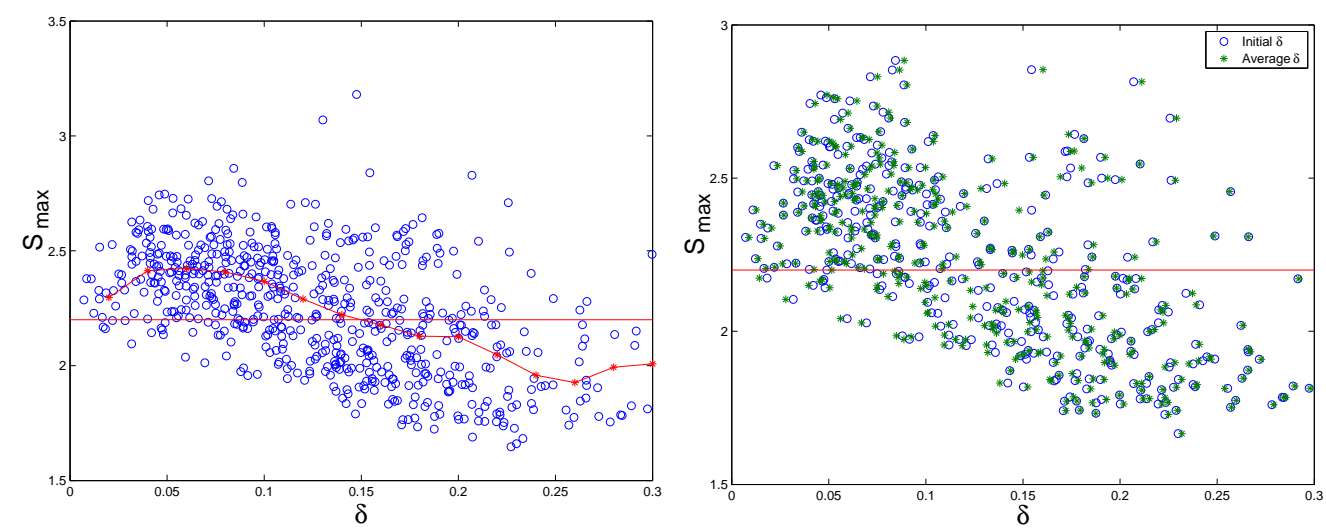

Figure 9: (left) NLS Bin Average and (right) $S_{\max }$ vs. $\delta_{0}$ (blue open circle) and $\delta_{\text {avg }}$ (green star) for the HONLS $(\epsilon=0.05)$.
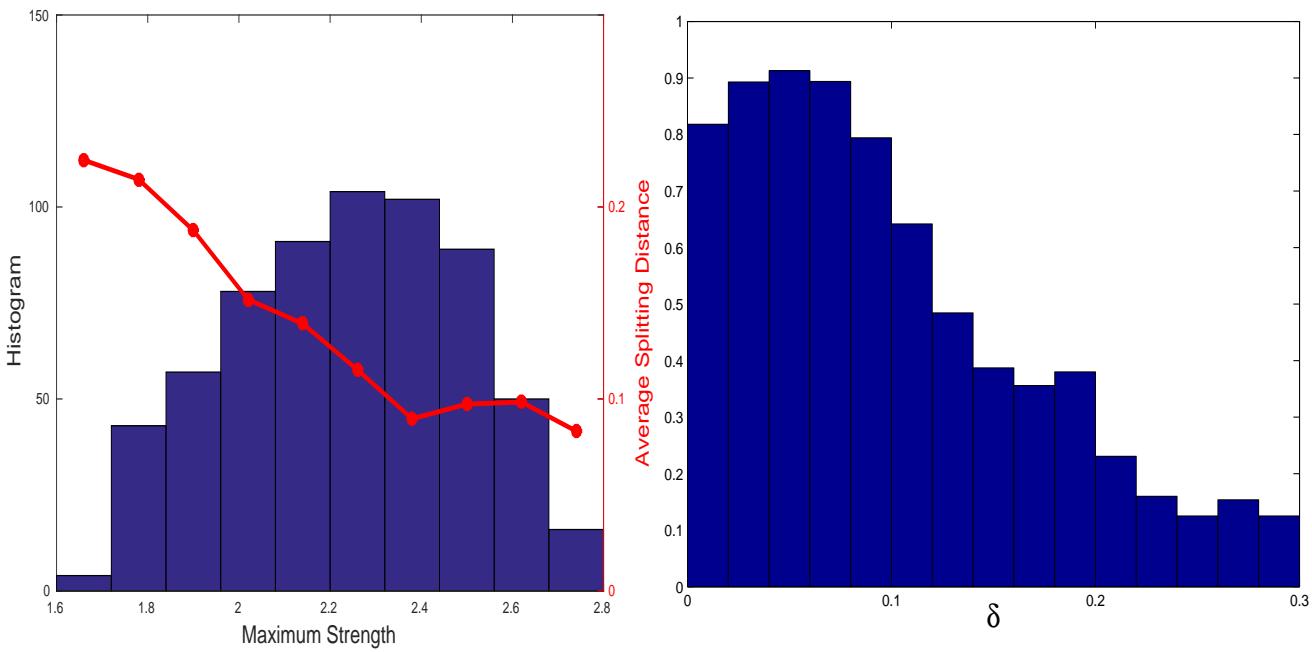

Figure 10: NLS experiments: (left) Maximum Strength Histogram (blue bars) and corresponding average splitting distance (red curve); (right) Probability $\mathcal{P}\left(\delta_{b i n}\right)$ of a rogue wave.

\subsection{HONLS experiments and the Floquet spectral quantities $\delta_{0}$ and $\delta_{\text {avg }}$}

The HONLS equation $(9, \Gamma=0)$ can be regarded as a perturbation of the NLS equation, and therefore we can interpret the resulting evolution in terms of the known dynamical properties of the NLS equation, i.e. in terms of the Floquet spectrum $\sigma(u)$, which is now no longer invariant. In particular, the splitting distance $\delta$ is a function of time, but in order to be able to use $\delta$ as a predictive tool, we would like to calculate it solely from the initial data. The question addressed below is whether $\delta(0)=\delta_{0}$ can be used for interpreting the HONLS dynamics in the context of rogue wave generation.

We observe that the time-average $\delta_{\text {avg }}=\operatorname{avg}_{t \in[0, T]} \delta(t)$ is an $\mathcal{O}(\epsilon)$-perturbation of $\delta_{0}$; therefore, for JONSWAP data, the dependence of $S_{\max }$ on $\delta_{\text {avg }}$ should not be significantly different from its dependence on $\delta_{0}$. This is confirmed in Figure (9)(right), showing $S_{\max }$ vs both $\delta_{0}$ and $\delta_{\text {avg }}$ for the HONLS experiments $(\epsilon=0.05, \Gamma=0)$ for an ensemble of JONSWAP initial data. As for the NLS experiments, $S_{\text {max }}$ decreases as $\delta$ increases. The probabilities $P\left(\delta_{b i n}\right)$ computed at $\delta_{0}$ and $\delta_{\text {avg }}$ are also very close (Figure (11)).

The HONLS experiments for JONSWAP data consistently show that $\delta_{0}$ remains a relevant diagnostic and that proximity to instabilities is still an important indicator of rogue wave events. This in turn implies that, also in the HONLS context, the heteroclinic orbits of 2-phase solutions of the NLS equation are appropriate models for JONSWAP rogue waves. 

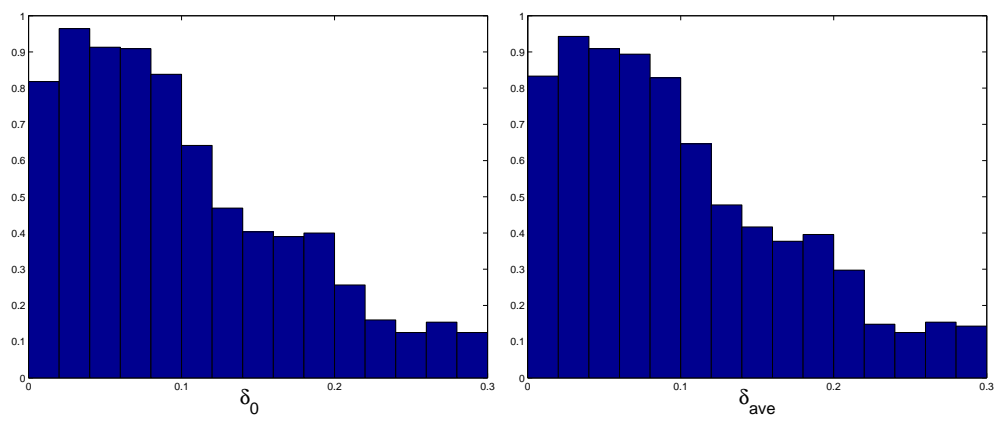

Figure 11: HONLS experiments: (left) probability $\mathcal{P}\left(\delta_{\text {bin }}\right)$ of a rogue wave using $\delta_{0}$ (left) and (right) using $\delta_{\text {avg }}$.

\subsection{Non-Gaussian behavior: comparison with field data}

In this section we examine whether the solutions of the NLS and HONLS equations for JONSWAP initial data yield non-Gaussian statistics in agreement with field studies of sea surface height variability.

In [24] Sura shows, empirically and theoretically, that the skewness Skew and the (excess) kurtosis $K$ associated with sea surface height obey the quadratic relation

$$
K=1.5(\text { Skew })^{2}-r
$$

where $r>0$ is an adjustment constant. This relation is not satisfied by e.g. a Gaussian or double exponential noise distribution. Figure (12) shows the skewness-kurtosis relation of data collected world-wide over a 10year span by the Ocean Topography Experiment (TOPEX)/Poseidon satellite altimeter. For comparison, the theoretical lower bounds for any PDF and unimodal PDF are given.

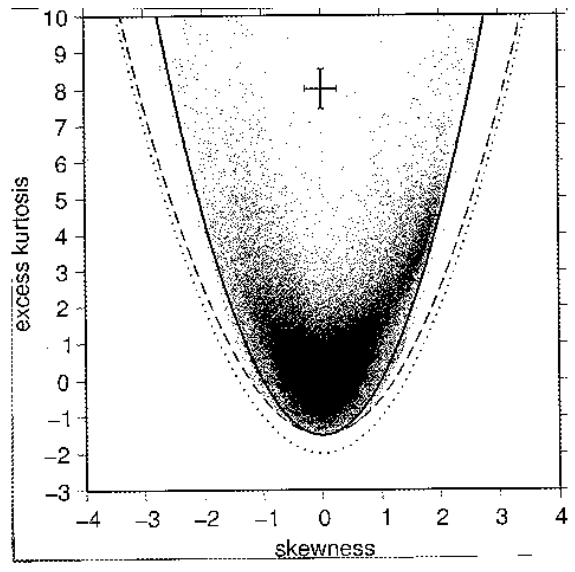

Figure 12: From Sura's paper [24], a comparison of skewness and kurtosis relations: $K \geq(\text { Skew) })^{2}-2$ for any PDF (dotted line), $K \geq(\text { Skew })^{2}-1.5$ for a unimodal PDF (dashed line), and $K \geq 3 / 2(\text { Skew })^{2}-1.5$ for SSH data (solid line).

This skewness-kurtosis relation is a first step in assessing whether a given model qualitatively describes observed sea surface height variability. Figure (13) shows the skewness-kurtosis plots for 10,000 random realizations of JONSWAP initial data at (left) $T=0$ and evolved to $T=10$ using the (middle) NLS equation and (right) HONLS equation with $\epsilon=0.05$. Clearly the quadratic skewness-kurtosis relation is satisfied by both the NLS and HONLS models. At $T=0$ the JONSWAP initial data appears Gaussian with a cluster of points above the vertex of the parabola. At $T=10$ the JONSWAP data develops into a distinctly non-Gaussian regime with the kurtosis achieving significantly larger values. An asymmetry develops in the area filled inside the parabola, similar to the observed asymmetry in the field data. The NLS and HONLS 
data are consistent with the TOPEX field data indicating that these equations are reasonable models for the SSH variability.
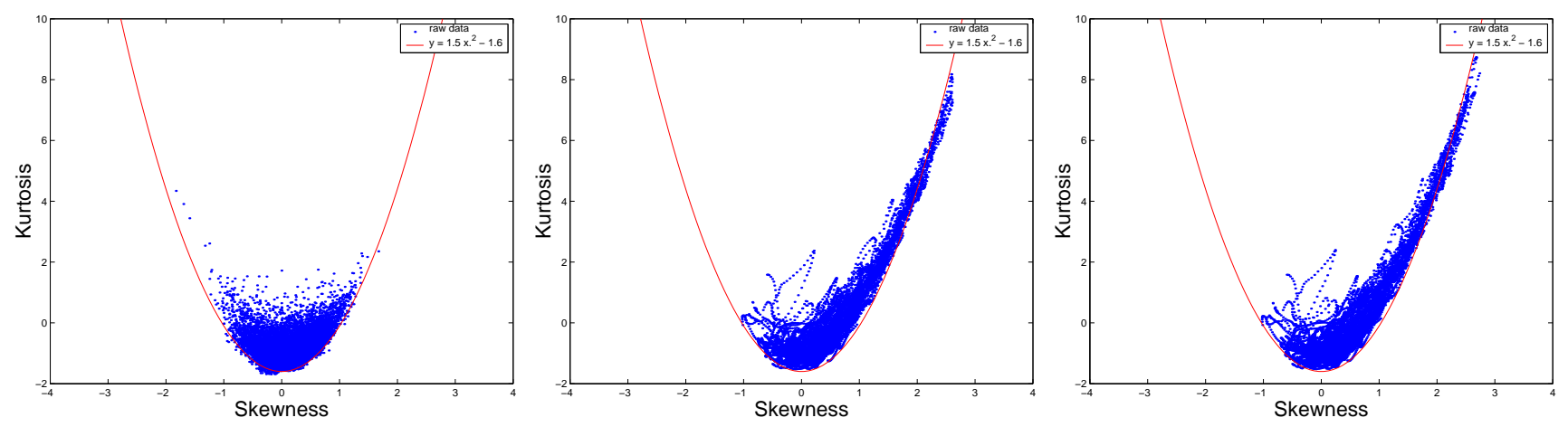

Figure 13: Skewness-kurtosis plots for: (left) JONSWAP initial data $(T=0)$, (middle) NLS solution evolved to $T=10$, and (right) HONLS solution evolved to $T=10(\epsilon=0.05)$. Solid line: $K=3 / 2(\text { Skew })^{2}-1.6$. The data also captures the asymmetric features of the plot.

To take a closer look at the non-Gaussian behavior of the data we bin the data for the HONLS experiments according to spectral gap size $\delta$ and consider the three bins: i) $\delta \leq .1$, ii) $.1<\delta<.2$, and iii) $\delta \geq .2$. The evolution of the excess kurtosis is presented in Figure 14(left) for these three ranges of $\delta$-values. The kurtosis is computed at each time step, first averaging over space and then averaging over the ensemble. Clearly the kurtosis attains larger asymptotic values for JONSWAP data closer to homoclinic data. As $\delta$ decreases the wave statistics change from almost-Gaussian type to distinctly non-Gaussian.

The over-prediction of the kurtosis and strength of rogue waves by the NLS and HONLS equations suggests including damping in the model. Figure (14)(right) refers to the damped HONLS experiments $(\epsilon=0.05$ and $0<\Gamma<0.04)$ for an ensemble of JONSWAP initial data. As in the NLS and HONLS experiments, $S_{\max }$ decreases with increasing $\delta$ (not shown). To get a handle on the effect of damping on rogue wave generation, we compute the critical value of $\delta$ such that $95 \%$ of the rogue waves occur for $\delta<\delta_{\text {critical }}$. Figure 14(right) shows that $\delta_{\text {critical }}$ significantly decreases as the damping parameter increases, indicating that the JONSWAP initial data must get significantly close to homoclinic data for rogue waves to occur. This indicates that proximity to homoclinic data is even more important for the development of rogue waves in the presence of damping.

This observation complements the results for the NLS and HONLS experiments and the use of heteroclinic orbits of N-phase solutions of the NLS equation to model rogue waves in JONSWAP sea states.
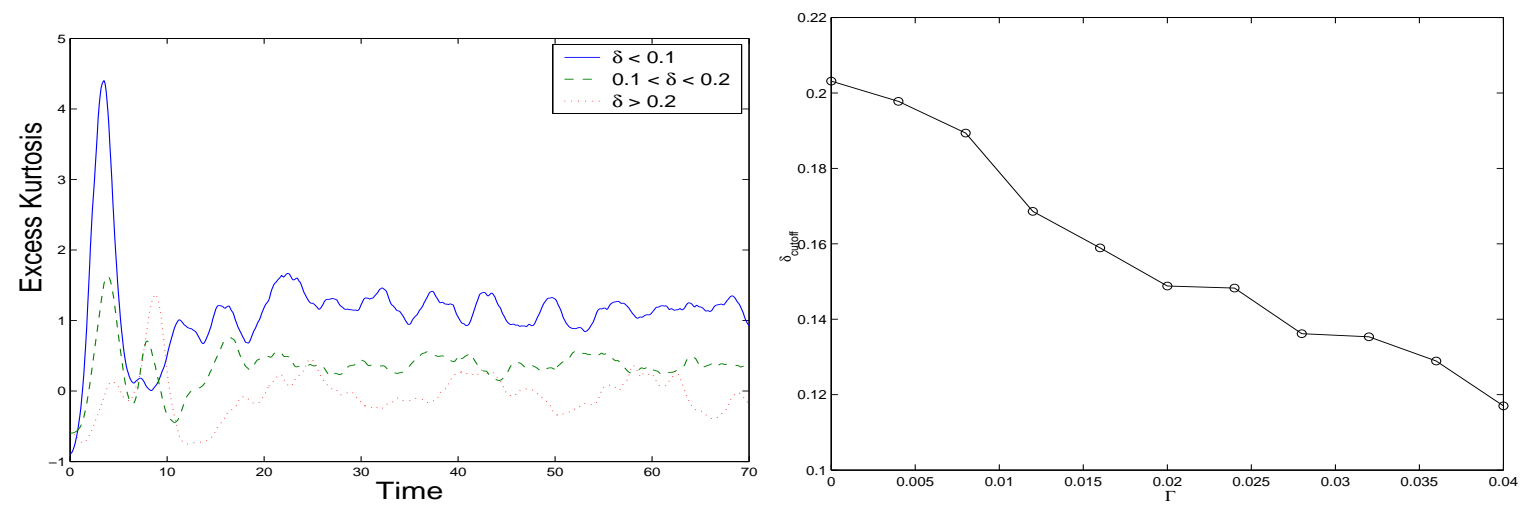

Figure 14: (left) HONLS experiments: dependence of averaged excess kurtosis on $\delta$, and (right) damped HONLS experiments $(\epsilon=0.05): \delta_{\text {critical }}$ as a function of $\Gamma$. 


\section{Conclusions}

We investigate extreme waves in random oceanic sea states characterized by JONSWAP power spectra using a nonlinear spectral decomposition provided by the integrable theory of the NLS equation over a periodic domain. We find that, for moderate values of the period, JONSWAP rogue waves can no longer be described by localized NLS solutions over a uniform background, but are appropriately modeled by heteroclinic orbits of 2-phase solutions of the NLS equation. We illustrate this finding by constructing heteroclinic orbits of certain modulationally unstable 2-phase solutions of the NLS equation by means of a Bäcklund transformation.

Using three approximate wave models, we show that the development of JONSWAP rogue waves is well predicted by proximity to homoclinic data of the NLS equation. All numerical experiments consistently demonstrate that the spectral diagnostic $\delta$ is an effective tool for determining the likelihood of rogue waves, and support the use of heteroclinic orbits of $\mathrm{N}$-phase solutions to model rogue waves in JONSWAP sea states.

We also show that proximity to homoclinic data generates a significant deviation from Gaussianity, and that the NLS and HONLS evolution of JONSWAP initial data yield non-Gaussian statistics in agreement with TOPEX field data. Thus the NLS and HONLS equations are reasonable models for sea surface height variability.

\section{Acknowledgements}

We wish to thank Brenton Lemesurier for his assistance with the numerical implementation of the Bäcklund transformation. Both authors gratefully acknowledge the support the National Science Foundation: A. Calini through grant DMS-1109017, and C.M. Schober through grant DMS-1108973.

\section{References}

[1] Akhmediev N, Ankiewicz A, and Soto-Crespo J.M. (2009) Rogue waves and rational solutions of the nonlinear Schrödinger equation. Phys. Rev. E 80 (2).

[2] Akhmediev N., Ankiewicz A. and Taki M. (2009) Waves that appear from nowhere and disappear without a trace. Phys. Lett. A 373, no. 6, 675.

[3] Ablowitz M.J., Kaup D.J., Newell A.C., Segur H. (1974) The inverse scattering transform-Fourier analysis for nonlinear problems. Stud. Appl. Math. 53, 249-315.

[4] Akhmediev N.N., Eleonskii V.M., Kulagin N.E., (1987) Exact First-Order Solutions of the Nonlinear Schrödinger Equation. Theor. and Math. Phys. (USSR) 72, no. 2, 809-818.

[5] Biondini G. and Mantzavinos D. (2016) Universal Nature of the Nonlinear Stage of Modulational Instability Phys. Rev. Lett. 116, 043902.

[6] Calini A, Schober C.M., (2002) Homoclinic chaos increases the likelihood of rogue waves. Phys. Lett. A 298, 335-349.

[7] Calini A, Schober C.M., (2009) Rogue Waves in Higher Order Nonlinear Schrödinger Models. In Extreme Ocean Waves (ed. E. Pelinovsky and C. Kharif), Springer. 31-51.

[8] Chabchoub A., Hoffmann N.P. and Akhmediev N. (2011) Rogue Wave Observation in a Water Wave Tank. Phys. Rev. Lett. 106, 204502.

[9] Chabchoub A. (2016) Tracking breather dynamics in irregular sea state conditions. Preprint ArXivv: $1604.06019 \mathrm{v} 1$. 
[10] Cox S.M. and Matthews P.C. (2002) Exponential time differencing for stiff systems. Journal of Computational Physics 176:430-455.

[11] Ercolani N., Forest M. G. and McLaughlin D. W. (1990) Geometry of the Modulational Instability Part III: Homoclinic Orbits for the Periodic Sine-Gordon Equation Physica D, 43, 349-384.

[12] Grimshaw R.H.J. and Tovbis A. (2013) Rogue waves: analytical predictions. Proc. R. Soc .A 469: 20130094.

[13] Islas A., Schober C.M. (2005) Predicting rogue waves in random oceanic sea states. Phys. Fluids 17:1-4.

[14] Janssen, P. (2004) The interaction of Ocean Waves and Wind Cambridge University Press.

[15] Li Y. and McLaughlin D.W., (1994) Morse and Melnikov functions for NLS Pde's. Comm. Math. Phys. 162, 175-214.

[16] Onorato M., Osborne A.R., Serio M. and Damiani T. (2001) Occurrence of freak waves from envelope equations in random ocean wave simulations, in Rogue Waves 2000, Olagnon, M. et al. (Ed.) Proceedings of a Workshop in Brest, France, 29-30 November 2000. Actes de Colloques - IFREMER, 32: pp. 181-191.

[17] Onorato M., Osborne A.R., Serio M. and Bertone S. (2001) Freak wave in random oceanic sea states. Phys. Rev. Lett. 86, 5831.

[18] Ochi M.K. (1998) Ocean waves: the stochastic approach. Cambridge University Press.

[19] Osborne A., Onorato M., Serio M. (2000) The nonlinear dynamics of rogue waves and holes in deep-water gravity wave trains. Phys. Lett. A 275:386.

[20] Osborne A.R. (2010) Nonlinear Ocean Waves 83 the Inverse Scattering Transform Vol. 97 (International Geophisics), Academic Press.

[21] Sergeeva A. and Slunyaev A. (2013) Rogue waves, rogue events and extreme wave kinematics in spatiotemporal fields of simulated sea states Nat. Hazards Earth Syst. Sci., 13, 1759-1771.

[22] Trulsen K., Dysthe K., (1996) A modified nonlinear Schrödinger equation for broader bandwidth gravity waves on deep water. Wave Motion 24, 281.

[23] Sattinger D.H. and Zurkowski V.D. (1987) Gauge theory of Bäcklund transformations. Physica D 26, $225-250$.

[24] Sura P. and S.T. Gille S.T. (2010) Stochastic dynamics of sea surface height variability J. Phys. Oceanogr. 40, 1592-1596. 